\title{
A Novel Approach based on Lifting Wavelet Transform for MIMO-OFDM Systems
}

\section{Elif Büşra Tuna}

Nuh Naci Yazgan Üniversitesi: Nuh Naci Yazgan Universitesi

Yusuf İslam Tek

Nuh Naci Yazgan Üniversitesi: Nuh Naci Yazgan Universitesi

Ali Ozen ( $\square$ aozen@nny.edu.tr)

Nuh Naci Yazgan University

\section{Research Article}

Keywords: MIMO-OFDM, fast Walsh Hadamard transform, Lifting wavelet transform, LWT-MIMO-OFDM, PAPR reduction

Posted Date: August 9th, 2021

DOl: https://doi.org/10.21203/rs.3.rs-751650/v1

License: (c) (i) This work is licensed under a Creative Commons Attribution 4.0 International License. Read Full License 


\title{
A Novel Approach based on Lifting Wavelet Transform for MIMO-OFDM Systems
}

\author{
Elif Büşra Tuna, Yusuf İslam Tek and Ali Özen* \\ Department of Electrical and Electronics Engineering \\ Nuh Naci Yazgan University - HARGEM \\ Kayseri, Turkey \\ elifbusratuna@gmail.com,yusuftek93@gmail.com, aozen@nny.edu.tr
${ }^{*}$ Correspondence to: Ali Özen, Nuh Naci Yazgan University - HARGEM, Department of Electrical and Electronics Engineering, Kayseri, Turkey. \\ E-mail: aozen@nny.edu.tr
}

\begin{abstract}
In this article, two methods are proposed to further increase the advantages of MIMO-OFDM systems such as high access quality, high data rates and spectral efficiency. The first of these is the combination of the MIMO-OFDM system with the fast Walsh Hadamard transform (FWHT) due to its high accomplishment with the ability to spread the data versus the disturbing influences of the channel. The second is the combination of Lifting wavelet transform (LWT), due to its superior advantages such as good time-frequency localization properties, ICI and ISI suppression capabilities due to its orthonormal structure, unlike fast Fourier transform (FFT), with MIMO-OFDM scheme. Computer simulation studies are carried out to verify the accomplishment of the suggested methods over the bit error rate (BER) and peak to average power ratio (PAPR) benchmark. From the acquired outcomes, it is noticed that approximately $6 \mathrm{~dB}$ of SNR gain and approximately $2 \mathrm{~dB}$ of PAPR gain are achieved with the proposed method.
\end{abstract}

Keywords-MIMO-OFDM; fast Walsh Hadamard transform; Lifting wavelet transform; LWT-MIMO-OFDM; PAPR reduction.

\section{INTRODUCTION}

Increasing spectral efficiency and improved connection security are the biggest challenges in the design of today's mobile and multi-user wireless communication systems. Increasing number of users and multimedia applications are increasing the need for high data rate in wireless communication systems. One of the problems encountered in achieving high data rates is multipath fading. The channel capacity of communication systems is significantly reduced due to the problems caused by multipath fading and inter user interference.

One of the methods to counter the fading effects in wireless communication systems is to use multi-antenna, in other words, multiple input multiple output (MIMO) systems. This method is known as antenna diversity [1]. In wireless communication systems, high access quality and high data rates can be achieved with the use of multiple antennas at the transmitter and receiver. MIMO systems are basically systems that enable to increase the capacity of the communication link without the need for additional transmit power and additional bandwidth by using multiple antenna arrays at the transmitter and receiver [2,3].

Orthogonal frequency division multiplexing (OFDM), on the other hand, is a multi-carrier modulation type that eliminates the increasing complexity due to frequency selective fading in radio channels and allows efficient use of the radio band [4]. With its multi-carrier structure orthogonal to each other, OFDM allows the data stream to be divided into parallel sub-taps and ensures that the channel exhibits flat fading characteristics in each tap. In this way, the effect of inter symbol interference (ISI) caused by fading is reduced and the error performance is increased [5]. In OFDM technique, high-speed data is divided to $K$ pieces low-speed data strings. Thus, the symbol duration is $K$ times longer (with the same symbol rate) compared to a system using a single carrier. The symbol duration becomes even longer with the addition of a cyclic prefix. Another important feature of OFDM is that it removes receiver complexity by allowing equalization in the frequency domain. In OFDM system using fast Fourier transform, broadband signal influenced by frequency selective channel is transformed into $K$ narrow band flat damping signals. Therefore, an equalization in the frequency domain is achieved with a scalar division [5].

MIMO systems have been used in OFDM systems, which allow high-speed data transmission in recent years, since they are more resistant to fading than single-input and single-output (SISO) systems. In this way, data losses that can be caused by fading in a MIMO-OFDM system are minimized and data transmissions with less loss and high gain are realized [6]. Since OFDM is suitable for multiple antennas and allows bandwidth, it also facilitates equalization in the MIMO technique it is used with. In this way, it is possible to reach high data rates with the effect of MIMO on the channel capacity, and with the multi-carrier feature of OFDM, the frequency selective feature of the frequency selective channel model is eliminated. Therefore, MIMO-OFDM forms the basis of the 
next generation of wireless communication. In recent years, the use of MIMO systems, in which multiple transmit and receive antennas are employed, together with OFDM modulation has gained popularity. So much so that MIMO-OFDM systems form the basis of 4th generation communication. This is because the two systems complement each other perfectly. MIMO systems, which were first proposed for the flat fading channel model, become practically usable with OFDM systems. The reason for this is that OFDM systems offer a solution for frequency selective fading, which is frequently encountered in real life and adversely affects the error performance. With their multi-carrier structure, they allow the information string to be split into parallel branches and for each sub-carrier, the channel presents flat fading. As employed with MIMO schemes, they can reach high data rates, which is a very important requirement in practice. The reason for this is that MIMO schemes heighten the channel capacity value [7].

However, OFDM systems are limited in use because of the precision in the positioning of sub-carriers and the consecutive pliability in frequency source allotment. These restrictions cause to more fascinating solutions in communication schemes employing novel waveforms based on versatile time and frequency filtering. Especially, the concern in communication systems employing novel waveforms that permit pliable utilize of time-frequency sources has raised in the last decade [8].

On the other hand, it is essential to have both time and frequency knowledge in information sending, particularly in non-static information. In such cases, the Fourier conversion is inadequate and the short time Fourier transform (STFT) can not simultaneously give a good time and good frequency localization. STFT can separately analyze the high frequency constituents with narrow windows (wideband frequency investigation) and the low frequency constituents with wide windows (narrow band investigation). However, since the static window function is employed in STFT, it is not possible to perform these inspections simultaneously. Therefore, a wavelet transform is needed, which gives good time localization at high frequencies and good frequency localization at low frequencies [9].

Although wave conversion has been used in signal processing applications for many years, it has been implemented beneficially in nearly every area of wireless communication technology in recent years $[10,11]$. Also, the basic principle in the OFDM waveform based on the wavelet transform is simply replacing the discrete Fourier transform (DFT) and inverse DFT (IDFT) with the discrete Wavelet transform (DWT) and inverse DWT (IDWT) respectively. DWT-based OFDM (DWT-OFDM) structure comes out as an alternative to FFT-based OFDM structure, because of the flexible structure of wavelet transform and adaptive time-frequency distances on subcarriers $[10,11]$. The separation of the input information to be sent in the DWT-OFDM scheme into distinctive frequency bands is executed by the low-pass and high-pass filter pairs.

In addition to these, orthogonal subcarriers in FFT-based OFDM schemes are comprised of sine/cosine signals. In such an application, the employ of rectangle window to distinguish subcarriers brings about high side band strength. In addition, the subcarriers are made up of the Sinc function, which has a boundless length in the frequency region. These two situations bring about ISI and inter carrier interference (ICI) drawbacks in FFT-based OFDM signals. These drawbacks can be mitigated by adding CP, which causes to a diminish in spectral efficiency. Thanks to the structural profit of wavelet-based OFDM techniques, there is no require to add CP. As an $X=\left[X_{0}, X_{1}, \ldots, X_{N-1}\right]$ data is made up of $N$ symbols are to be sent over the communication channel, each symbol of this signal is sent with $N$ subcarriers with equal band-widths in the FFT-OFDM scheme. On the other hand, in the DWTOFDM scheme, each symbol has the occasion to be sent with sub-carriers with different band-widths. This flexibility enables the performance of the schemes to be improved by adapting the subcarrier scheme of DWT-OFDM in accord with the condition of the communication surroundings. The most basic reason for using DWT in the multi-carrier communication system structure is that it is based on orthogonality. Wavelets can be planned to accomplish better frequency localization and can eliminate the need for guard intervals by overlapping symbols in the time domain. This overlapping attribute raises symbol duration and thus provides better spectral efficiency. Also, the use of wavelet filters provides greater pliability, suggests a higher degree of stopband weakening, and results in good side lobe suppression [12]. Thus, the spectral loss caused by the use of CP in FFT-OFDM systems is reduced by the DWT method. In addition, in DWT-based multi-carrier communication system structures, the inverse wavelet transform output has a lower variance compared to the inverse Fourier transform output in OFDM [12]. As a result of this, it means that lower PAPR can be accomplished in DWT-based multi-carrier communication systems.

However, the filters employed in conventional DWT processes include complex mathematical processes, and the Lifting wavelet transform (LWT) scheme has been improved to decrease this. LWT, which has been employed in image and signal processing implementations in recent years, is employed to mitigate many deficiencies of classical wavelet transform. The LWT is intended to decrease the working burden in the wavelet transform and alter the DWT employed in the computation of the wavelet coefficients [12]. Lifting scheme is the simplest and most efficient technique for wavelet transform [13]. When compared FFT and DWT with LWT, LWT's

- Reduced computational complexity,

- Compared with the filter bank structure, better frequency localization and power consumption,

- Easy implementation on the hardware side due to significantly reduced computational complexity,

has such advantages.

In this developed study, inspired by [8, 9, 14], it is suggested to combine MIMO-OFDM method with LWT conversion to improve both BER accomplishments and PAPR accomplishments in MIMO-OFDM communication schemes. Thus, it can be 
observed from the computer simulation outcomes that remarkable performances are acquired in both PAPR achievements and BER achievements in wireless communication schemes where LWT-MIMO-OFDM technique is applied. Even though there are a few studies [15-17] on the DWT-MIMO-OFDM system for PAPR reduction in the literature, as far as the authors know, there has been no study investigating MIMO-OFDM communication systems by combining MIMO-OFDM and LWT.

In this study, another proposed method to increase the performance of MIMO-OFDM systems is to combine the MIMO-OFDM system with fast Walsh Hadamard transform (FWHT) due to its high achievement with the ability to spread data against the channel's disturbance effects.

High reliability is evaluated by the bit error rate (BER) and is highly significant for systems where errors are less endurable. At high energy efficiency, low computational burden and low peak to average power ratio (PAPR) provide high energy efficiency [8, 9, 14]. For these reasons, BER and PAPR changes were used as benchmark criterion in our analyzes. In addition to these, with the simulation outcomes, it has been demonstrated that the recommended LWT-MIMO-OFDM structure has lower PAPR and BER performances than conventional MIMO-OFDM schemes in areas where the distortion influences of the MIMO channel are efficient.

The remaining of the paper is arranged as below; Section 2 summarizes the general structure of the MIMO-OFDM communication scheme. In Chapter 3, explanations about the LWT-based MIMO-OFDM structure are given. Finally, computer simulation outcomes, inferences and discussions are presented in Chapters 4 and 5.

\section{MIMO-OFDM SYSTEMS}

MIMO-OFDM systems are obtained as a result of the use of multiple antenna systems in the receiver and transmitter layers to improve the system capacity on time-varying or frequency-selective channels and to increase the diversity gain of an OFDM system. A block diagram of a MIMO-OFDM system is given in Figure 1.

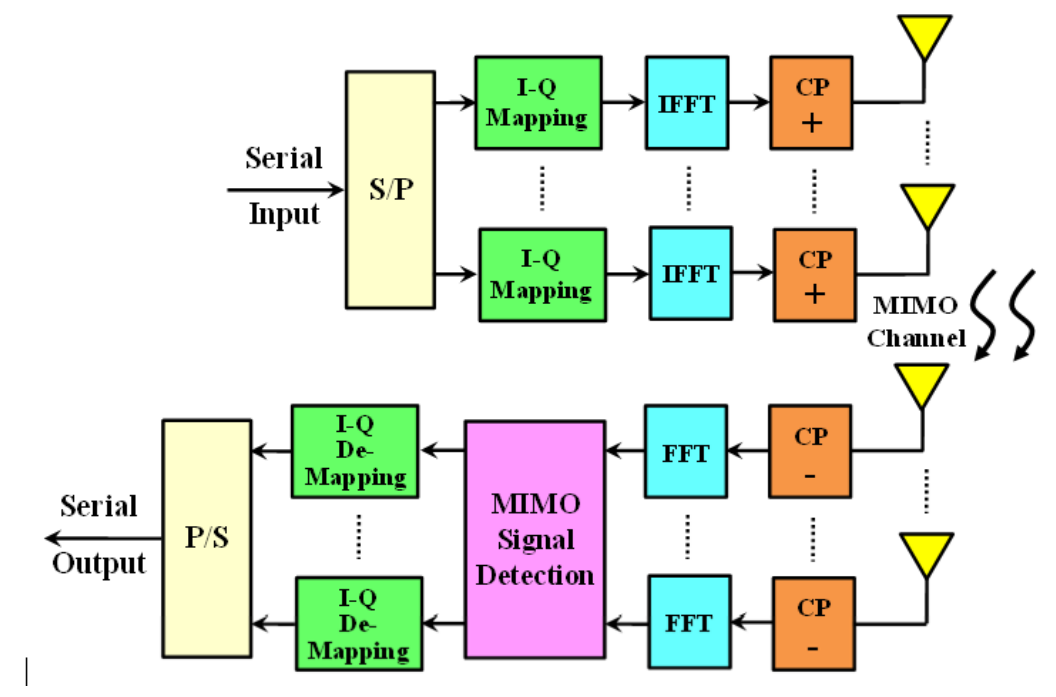

Fig. 1. Block diagram of MIMO-OFDM scheme.

The MIMO-OFDM scheme shown in Figure 1 is composed of $M$ transmitting and $N$ receiving antennas. Random binary data coming from the input of the system is modulated after being grouped depending on the modulation type to be used. Then the modulated data is separated depending on the number of antennas in the system. There is a single-input, single-output OFDM structure in the arm connected to each antenna. In each OFDM structure, the serial symbol stream is converted to parallel so that the IFFT can be received. The IFFT process provides the generation of subcarriers and conversion from the frequency region to the time region in OFDM. Later, CP is added to prevent inter-symbol interference, and then symbols are transmitted serially in the wireless environment. After the symbols sent from the $m$ th transmitting antenna at the $n$th receiving antenna are converted into parallel, the CP is removed and the following signal is obtained after receiving its FFT. Thus, the MIMO-OFDM signal for the $k$ th subcarrier and the $n$th receiving antenna can be written as follows:

$$
Y_{n}[k]=\sum_{m=1}^{M} X_{m}[k] H_{n, m}[k]+W_{n}[k]
$$


Here, $X_{m}[k]$ is the OFDM data symbol transmitted with the $m$ th transmit antenna and the $k$ th subcarrier $\left(k=0,1, \ldots K_{-1}\right), W_{n}[k]$ corresponding to the symbol in the frequency region of the $n$th antenna $\sigma_{n}^{2}$ variance and zero mean additive white Gaussian noise, $H_{n, m}[k]$, as given in the following equation, in the $k$ th frequency region $n$th receiver $(n=1,2, \ldots N)$ and $m$ th transmitter $(m=1,2$, $\ldots, M)$ are the channel coefficients between the antennas.

$$
H_{n, m}[k]=\sum_{l=0}^{L-1} h_{n, m}[l] e^{-j 2 \pi l k / K}
$$

Here, the $h$ 's are modeled as a zero-mean independent Gaussian random process. The impulse response of the Rayleigh fading channel can be written as:

$$
h(t, \tau)=\sum_{l=0}^{L-1} h_{l}(t) \delta\left(\tau-\tau_{l}(t)\right)
$$

The channel impulse response is supposed to be stationary over the OFDM channel symbol time. $T_{\text {channel }}=T_{S}+T_{C P}$; where $T_{C P}$ is the cyclic prefix time, $T_{S}$ is the symbol time. This corresponds to a slowly changing channel; that is, the coherence time is greater than the symbol time. This supposition impedes the occurrence of inter-carrier interference (ICI).

The $H$ channel matrix is also a $N \times M$ matrix, for $N$ receiving $(n=1,2, \ldots, N)$ and $M$ transmitting $(m=1,2, \ldots, M)$ antennas $(n, m)$ th components can be written as:

$$
H[k]=\left[\begin{array}{ccc}
H_{1,1}[k] & \cdots & H_{1, M}[k] \\
\vdots & \ddots & \vdots \\
H_{N, 1}[k] & \cdots & H_{N, M}[k]
\end{array}\right]
$$

The received information at the receiving side can be represented in matrix form as follows:

$$
Y[k]=X[k] H[k]+W[k]
$$

Here, $\boldsymbol{X}[\boldsymbol{k}]=\left[X_{1}[k] X_{2}[k] \cdots X_{M}[k]\right]^{T}$ and $\boldsymbol{Y}[\boldsymbol{k}]=\left[Y_{1}[k] Y_{2}[k] \cdots Y_{N}[k]\right]^{T}, \quad M \times 1$ and $N \times 1$ vectors consist of transmitted and received data.

\section{Proposed LWT-MIMO-OFDM SYSTEMS}

In this developed study, inspired by [8, 9, 14], the unit scheme of the recommended LWT-MIMO-OFDM technique, which combines MIMO-OFDM communication systems and LWT conversion, is shown in Figure 2. 


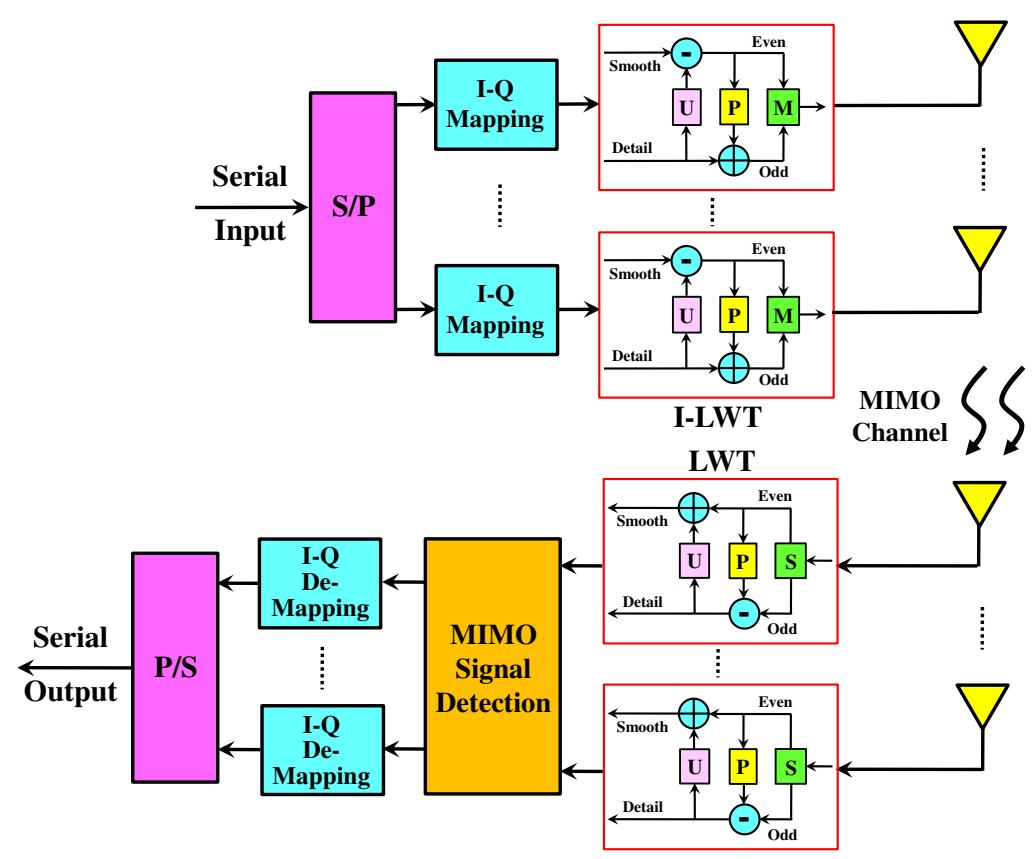

Fig. 2. Block diagram of the proposed LWT-MIMO-OFDM systems.

On the transmitter region of the LWT-MIMO-OFDM scheme illustrated in Fig. 2, the modulated data is used in a single-input, single-output OFDM structure in each branch connected to the antenna. In the proposed method, ILWT transformation is enforced to the modulated data with the aid of the following formula instead of the IFFT process in each OFDM structure (for detailed information on ILWT/LWT, see [8, 9, 14]).

$$
V_{I L W T}[k]=\sum_{n=0}^{\infty} \sum_{z=0}^{U-1} X^{n}[z] 2^{\frac{n}{2}} \varphi\left[2^{n} k-z\right]
$$

Here, $X^{n}[k]$ is the modulated data, $U$ is the number of subcarriers, and $\varphi[k]$ is the wavelet function for the $z$ th-order sub-carrier. The data packets produced after the ILWT conversion are sent through the frequency selective Rayleigh fading channel via antennas and arrive to the receiver later being distorted by the additive white Gaussian noise (AWGN). On the receiving side, the opposite of the transactions on the transmitter part is applied. On the receiving part, LWT conversion is applied to the data packets received from each antenna with the aid of the below formula instead of the FFT process in the classical OFDM structure.

$$
Y_{L W T}^{Z}[k]=\sum_{n=0}^{N-1} R[n] 2^{\frac{k}{2}} \varphi\left[2^{k} n-z\right], k=0,1,2, \ldots, N-1, z=0,1,2, \ldots, N-1
$$

Here, $R[n]$ denotes the degraded information with frequency selective channel and AWGN. The acquired information at the output of the LWT Unit is compensated in the MIMO Signal Detection Block. A flat channel equalizer is used in the MIMO Signal Detection Block, assuming that the MIMO channel is perfectly estimated. Then, after the corrected data is de-modulated in the I-Q De-Mapping Unit, the requested accomplishment criteria are attained.

In this study, it is recommended to combine FWHT transform and MIMO-OFDM system to examine the effects of FWHT conversion on the accomplishment of MIMO-OFDM schemes. On the transmitter part of the MIMO-OFDM scheme presented in Fig. 1, FWHT conversion is enforced to the modulated data with the aid of the presented following formula.

$$
V_{F W H T}[k]=\sum_{n=0}^{U-1} X[n] F W[n, k], \quad k=0,1, \ldots, U-1
$$


Here, $F W[n, k]$ denotes the FWHT transformation. Then, the IFFT transformation is applied to the data to which the FWHT transformation is enforced, with the assist of the following equality.

$$
V_{I F F T}[n]=\frac{1}{\sqrt{N}} \sum_{u=0}^{U-1} V_{F W H T}[u] e^{\frac{j 2 \pi u n}{U}}, \quad 0 \leq n \leq U-1
$$

Then, the above-described processes are applied here as well, and after $\mathrm{CP}$ is removed from the data packets reaching the receiver as corrupted, FFT process is enforced with the assist of the following equality.

$$
Y_{F F T}[k]=\sum_{i=0}^{U-1} R[i] e^{\frac{-j 2 \pi k i}{U}}, \quad k=0,1, \ldots, U-1
$$

After that, with the assist of the following equality, inverse FWHT (IFWHT) transformation is enforced to the data in the frequency domain.

$$
Y_{I F W H T}[k]=\frac{1}{U} \sum_{\mathrm{n}=0}^{U-1} Y_{F F T}[n] F W[n, k], \quad k=0,1, \ldots, U-1
$$

Finally, the above processes are repeated here, and the requested accomplishment criteria are computed by employing the obtained data at the output of the I-Q De-Mapping Unit.

\section{Computer Simulation Studies}

Computer simulation works are comprised of two chapter, BER-SNR performances in flat Rayleigh fading channel environment and PAPR performances. In the simulations, the BER and PAPR accomplishments of the Classic MIMO-OFDM, the proposed FWHT-MIMO-OFDM and the suggested LWT-MIMO-OFDM systems are compared in the $2 \times 2$ MIMO-OFDM scheme, in which 2 transmit antennas and 2 receive antennas are used. Computer simulations are acquired with 1000 Monte Carlo channel loops for BPSK, 4-QAM, 16-QAM and 64-QAM modulations. All of the simulation works were performed employing 1000 MIMO-OFDM data packets, 16-bit CP, 64 subcarriers and 64-point FFT operation [18].

\section{A. Flat Rayleigh Fading Channel Simulation Results}

In the first chapter, in which BER-SNR simulation works are carried out in the flat Rayleigh fading channel surroundings, classical MIMO-OFDM and the proposed FWHT-MIMO-OFDM and proposed LWT-MIMO-OFDM systems are compared. BERSNR performances are acquired for BPSK and 4-QAM signals.

The impacts of distinctive wave families for BPSK modulation on the BER accomplishment of the suggested LWT-MIMOOFDM system are shown in Figure 3. 


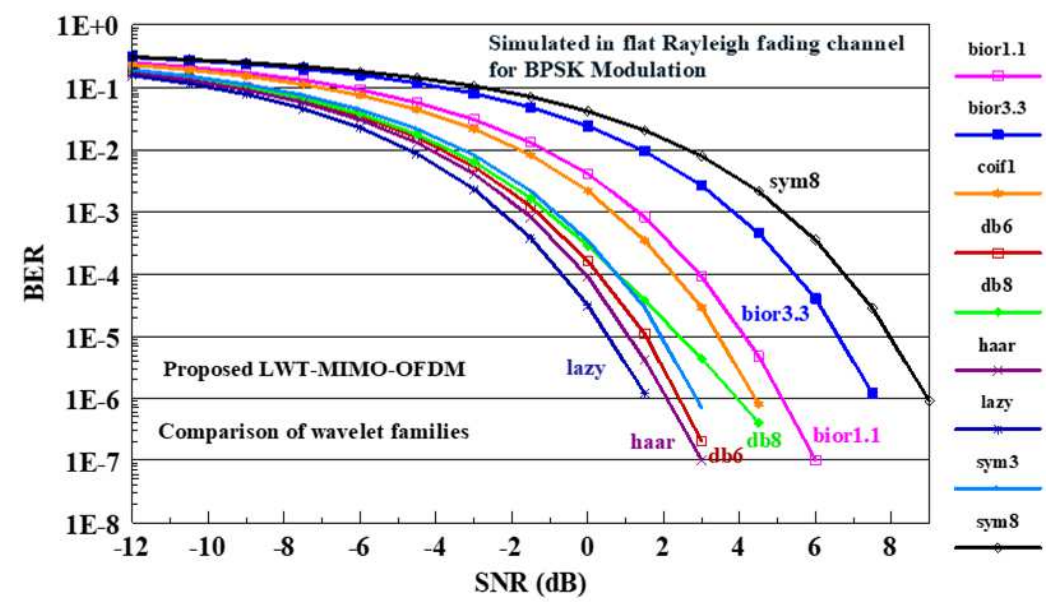

Fig. 3. The acquired BER performances of the offered LWT-MIMO-OFDM technique with distinctive wave families in flat Rayleigh fading channel for BPSK signal.

In Fig. 3, as the effects of distinctive wave families such as bi-orthogonal1.1, bi-orthogonal3.3, coiflets1, daubechies6, daubechies8, haar, lazy, symlets3 and symlets8 on the acquired BER accomplishment of the offered LWT-MIMO-OFDM system are inspected in the flat Rayleigh fading channel for BPSK signal, it is seen that the best accomplishment is obtained with the lazy wave family and the worst accomplishment is obtained with the symlets8 wave family. The accomplishment of other wave families tumbles between the performances of these two wave families. Since the best accomplishment is obtained with the lazy wave family in this simulation, the lazy wave family is used in the suggested LWT-MIMO-OFDM system in the next comparison.

The BER performances of classical MIMO-OFDM, proposed FWHT-MIMO-OFDM and suggested LWT-MIMO-OFDM systems are compared for BPSK signal in Figure 4.

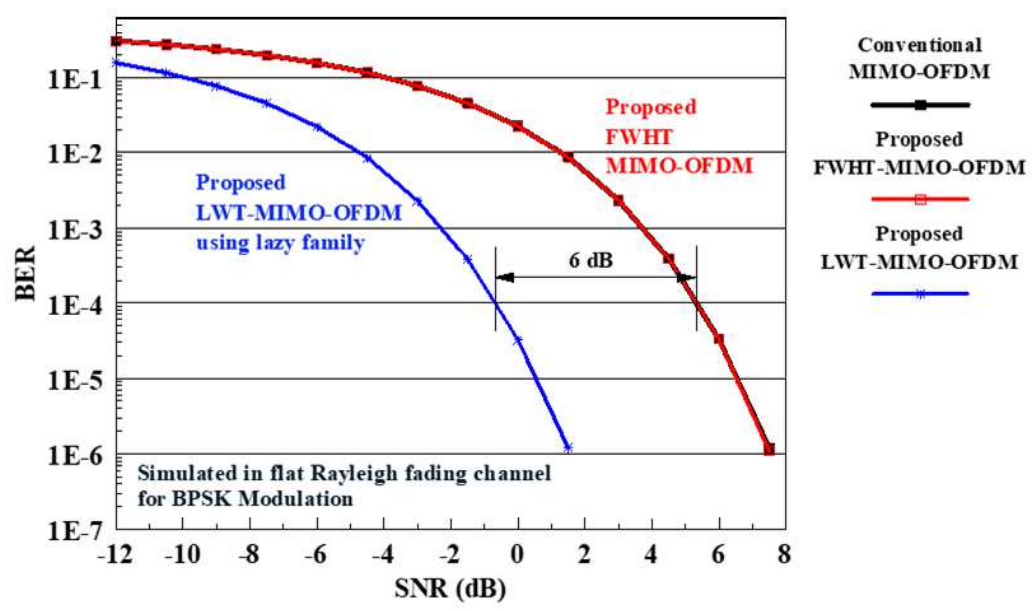

Fig. 4. Evaluation of BER-SNR accomplishments of traditional MIMO-OFDM, offered FWHT-MIMO-OFDM and offered LWT-MIMO-OFDM systems in flat Rayleigh fading channel for BPSK modulation.

From Figure 4, where the BER-SNR performances are evaluated, it is noticed that the suggested FWHT transform has no assistance to the BER performance of MIMO-OFDM systems in the flat Rayleigh fading channel environment. The performances of the classical MIMO-OFDM and the proposed FWHT-MIMO-OFDM systems are almost the same. However, it is perceived that the proposed LWT-MIMO-OFDM scheme surpasses both methods and gives roughly $6 \mathrm{~dB}$ SNR development for 1E-4 BER level.

The effects of different wavelet families for 4-QAM modulation on the BER accomplishment of the offered LWT-MIMOOFDM technique are illustrated in Fig. 5. 


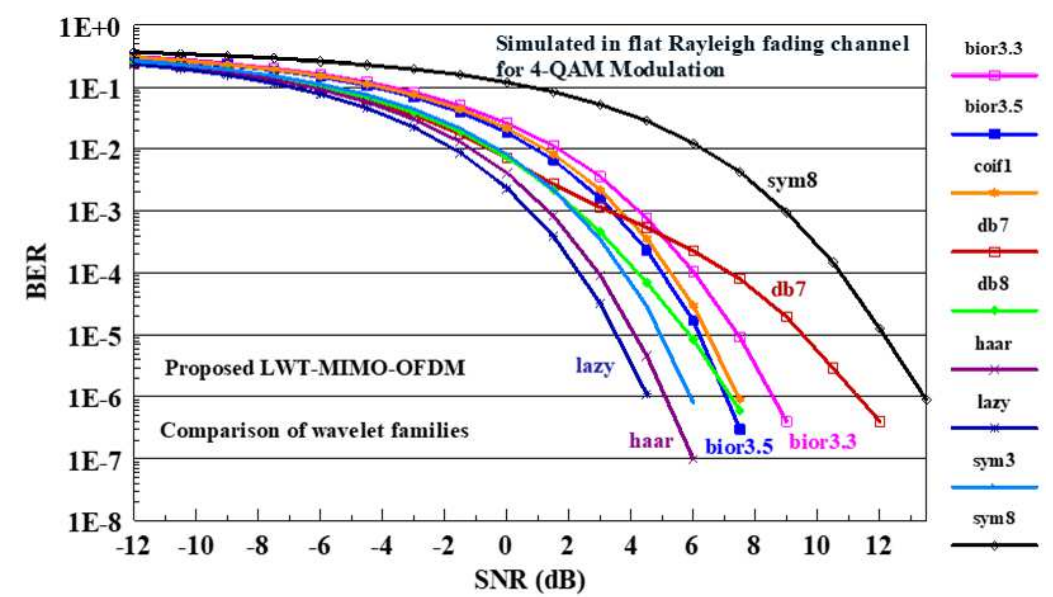

Fig. 5. The acquired BER accomplishments of the offered LWT-MIMO-OFDM method with distinctive wave families in flat Rayleigh fading channel for 4-QAM signal.

As Fig. 5 is examined, it is noticed that the best accomplishment is attained with the lazy wave family, similar to the previous BPSK modulation. Therefore, as the best accomplishment is attained with the lazy wave family in this simulation, the lazy wave family is employed in the offered LWT-MIMO-OFDM system in the next evaluation.

The BER accomplishments of traditional MIMO-OFDM, suggested FWHT-MIMO-OFDM and offered LWT-MIMO-OFDM systems are compared for 4-QAM signal in Figure 6.
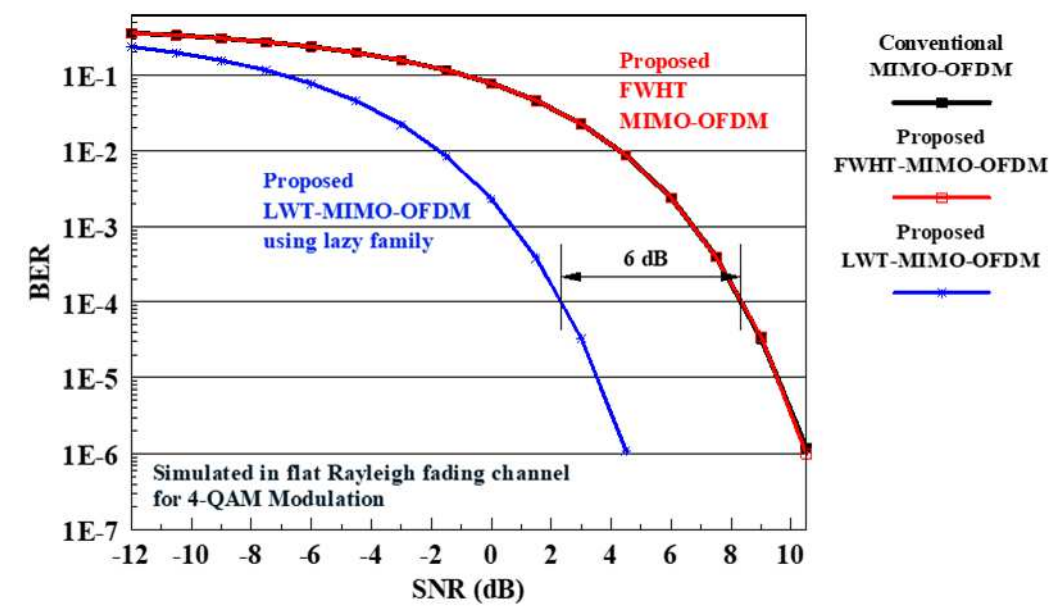

LWT-MIMO-OFDM

Fig. 6. Evaluation of BER-SNR accomplishments of traditional MIMO-OFDM, offered FWHT-MIMO-OFDM and offered LWT-MIMO-OFDM systems in flat Rayleigh fading channel for 4-QAM signal.

As Fig. 6 is analyzed, it is understood that similar outcomes and gains to the prior BPSK modulation were obtained. It is observed from Figure 6 that there is an increase in SNR values as only the degree of modulation increases.

\section{B. PAPR Simulation Results}

In the 2nd chapter, in which PAPR simulation works are performed, classical MIMO-OFDM and proposed FWHT-MIMOOFDM and proposed LWT-MIMO-OFDM systems are compared. PAPR performances are obtained for 16-QAM and 64-QAM modulations.

Since each antenna has a SISO-OFDM signal in MIMO-OFDM systems, a discrete-time OFDM signal can be expressed mathematically as in the formula below.

$$
x[n]=\frac{1}{\sqrt{U}} \sum_{k=0}^{U-1} X[k] e^{\frac{j 2 \pi k n}{U}}, \quad 0 \leq k \leq U-1
$$


Here, $n$ are discrete sampling points. $X[k]$ is the modulated data and $U$ is the number of subcarriers. Equation (12) expresses the inverse discrete Fourier transform (IDFT).

For calculating the actual PAPR value of the continuous time OFDM signal in practical applications, in a discrete time OFDM signal; By adding $(L-1) U$ zeros to the discrete-time $x[n]$ OFDM signal, $L$ times upsampling is applied. The signal $x[n]$, consisting of the IFFT point of length $L U$, can be described as:

$$
x[n]=\sum_{k=0}^{L U-1} X[k] e^{\frac{j 2 \pi k n}{L U}}, \quad 0 \leq n \leq L U-1
$$

Thus, the PAPR value of an OFDM signal can be defined as:

$$
\operatorname{PAPR}(x[n])=10 \log _{10}\left[\frac{\max _{0 \leq n \leq L U-1}\left\{|x[n]|^{2}\right\}}{E\left\{|x[n]|^{2}\right\}}\right]
$$

Here, $\max [$.$] is the peak power of the signal, and E[$.$] is the expected value of the signal.$

In this study, 2 antennas are used in the transmitter for $\boldsymbol{X}[\boldsymbol{k}]=\left[X_{1}[k] X_{2}[k] \cdots X_{M}[k]\right]^{T}$ input signal in MIMO-OFDM system. Therefore, time domain $x_{1}[n]$ and $x_{2}[n]$ signals are obtained by applying IFFT to $X_{1}[k]$ and $X_{2}[k]$ signals produced for 2 antennas. Thus, the PAPR value in the MIMO-OFDM scheme can be expressed as follows [19]:

$$
P A P R_{M I M O}=\max \left\{P A P R\left(x_{1}[n]\right), P A P R\left(x_{2}[n]\right)\right\}
$$

The success of a PAPR reduction method is evaluated by the Complementary Cumulative Distribution Function (CCDF) measurement criterion. This value can be described as:

$$
\operatorname{CCDF}[\operatorname{PAPR}(x[n])]=\operatorname{Pr}[\operatorname{PAPR}(x[n]>\delta)]
$$

Here, $\delta$ represents a certain threshold level and $C C D F[$.$] depicts the probability that the PAPR value of the x[n]$ signal is higher than the $\delta$ threshold level.

The impacts of distinctive wave families on the PAPR accomplishment of the offered LWT-MIMO-OFDM system are shown for 16-QAM signal in Figure 7. 


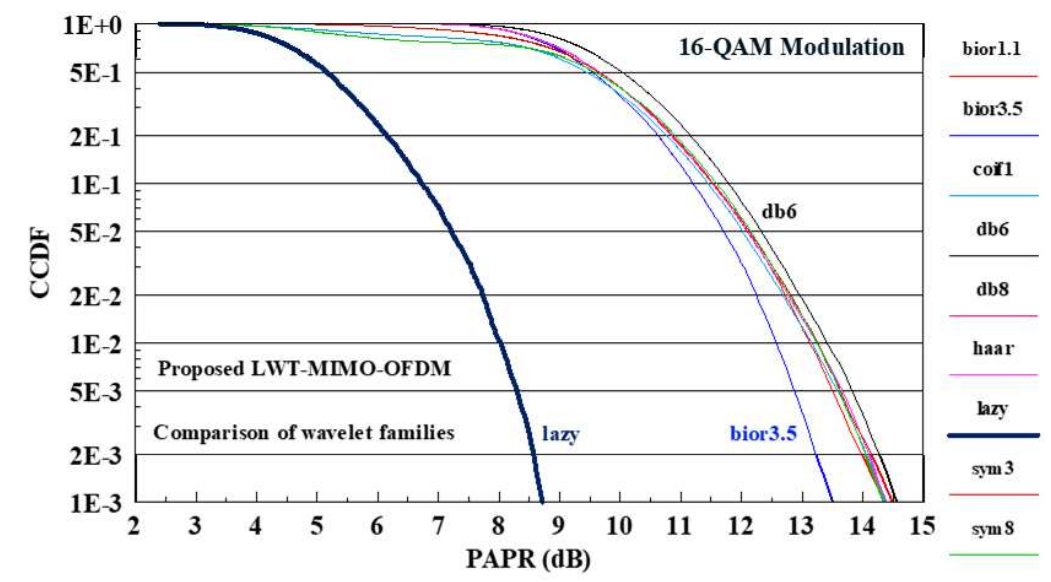

Fig. 7. The obtained PAPR accomplishments of the offered LWT-MIMO-OFDM technique with distinctive wave families for 16-QAM signal.

When Fig. 7 is inspected, the impacts of distinctive wave families such as bi-orthogonal1.1, bi-orthogonal3.5, coiflets1, daubechies6, daubechies8, haar, lazy, symlets3 and symlets8 on the acquired PAPR performance of the proposed LWT-MIMOOFDM system for 16-QAM signal, it is seen that the best accomplishment is acquired with the lazy wave family and the worst accomplishment is obtained with the daubechies6 wave family. The performance of other wave families falls between the performances of these two wave families. Since the best accomplishment is obtained with the lazy wave family in this simulation, the lazy wave family is used in the suggested LWT-MIMO-OFDM system in the next comparison.

The PAPR performances of classical MIMO-OFDM, proposed FWHT-MIMO-OFDM and proposed LWT-MIMO-OFDM methods are evaluated in Fig. 8 for 16-QAM signal.

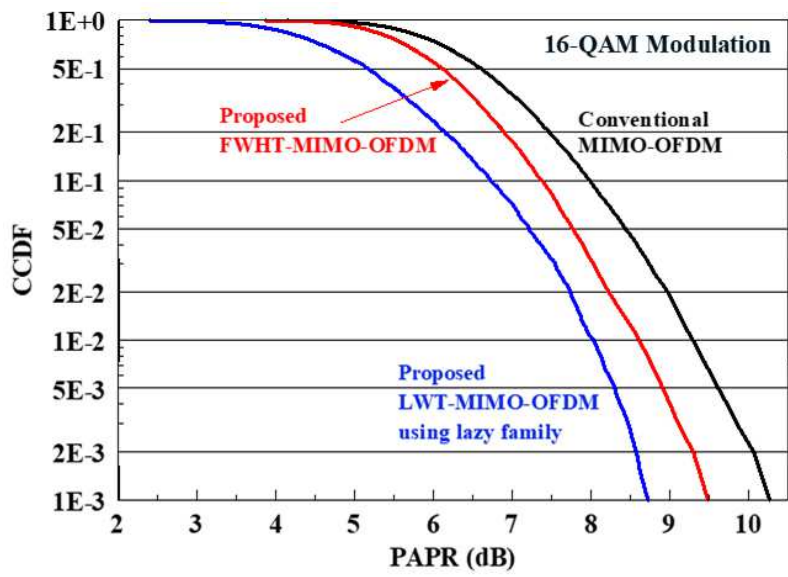

$$
\begin{aligned}
& \text { Conventional } \\
& \text { MIMO-OFDM } \\
& \text { Proposed } \\
& \text { FWHT-MIMO-OFDM }
\end{aligned}
$$

Fig. 8. Evaluation of PAPR accomplishments of classic MIMO-OFDM, suggested FWHT-MIMO-OFDM and recommended LWT-MIMO-OFDM systems for 16-QAM signal.

From Figure 8, where PAPR performances are analyzed, it is noticed that although the suggested FWHT conversion has no contribution to the BER performance of MIMO-OFDM systems, it surprisingly provides a PAPR development of roughly $1 \mathrm{~dB}$ for 1E-3 PAPR level versus MIMO-OFDM systems. However, it is noticed that the best PAPR accomplishment is acquired with the proposed LWT-MIMO-OFDM scheme and approximately $1.8 \mathrm{~dB}$ PAPR development is achieved for 1E-3 PAPR level compared to the MIMO-OFDM system.

The impacts of distinctive wave families on the PAPR accomplishment of the suggested LWT-MIMO-OFDM system are demonstrated in Fig. 9 for 64-QAM signal. 


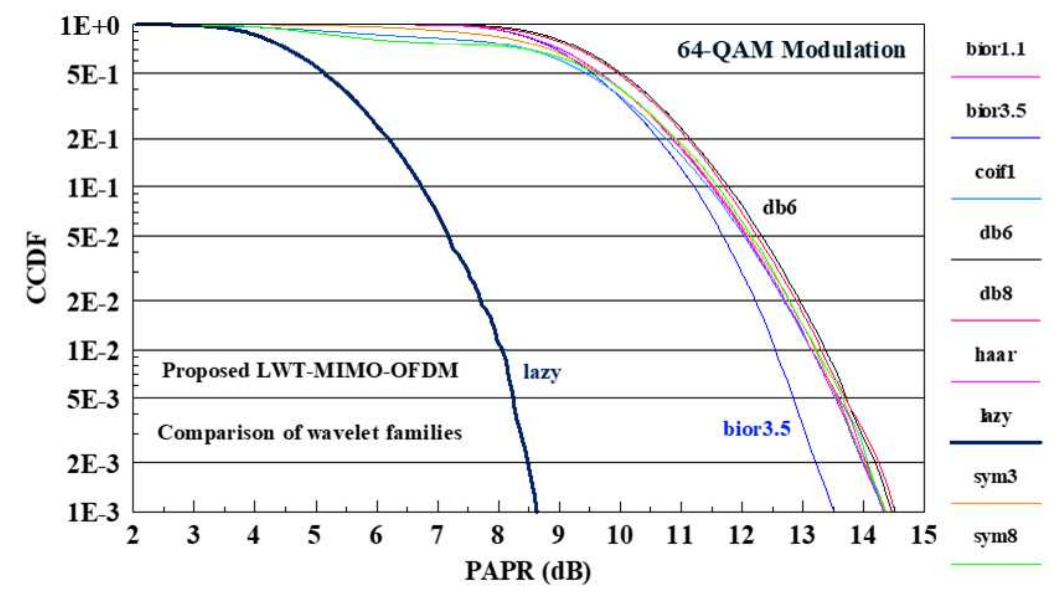

Fig. 9. The obtained PAPR accomplishments of the offered LWT-MIMO-OFDM technique with distinctive wave families for 64-QAM signal.

As Fig. 9 is ispected, it is noticed that the best accomplishment is attained with the lazy wave family, similar to the previous 16QAM modulation. Therefore, since the best accomplishment is obtained with the lazy wave family in this simulation, the lazy wave family is used in the suggested LWT-MIMO-OFDM system in the next comparison.

The PAPR performances of classical MIMO-OFDM, proposed FWHT-MIMO-OFDM and proposed LWT-MIMO-OFDM systems are compared in Figure 10 for 64-QAM modulation.
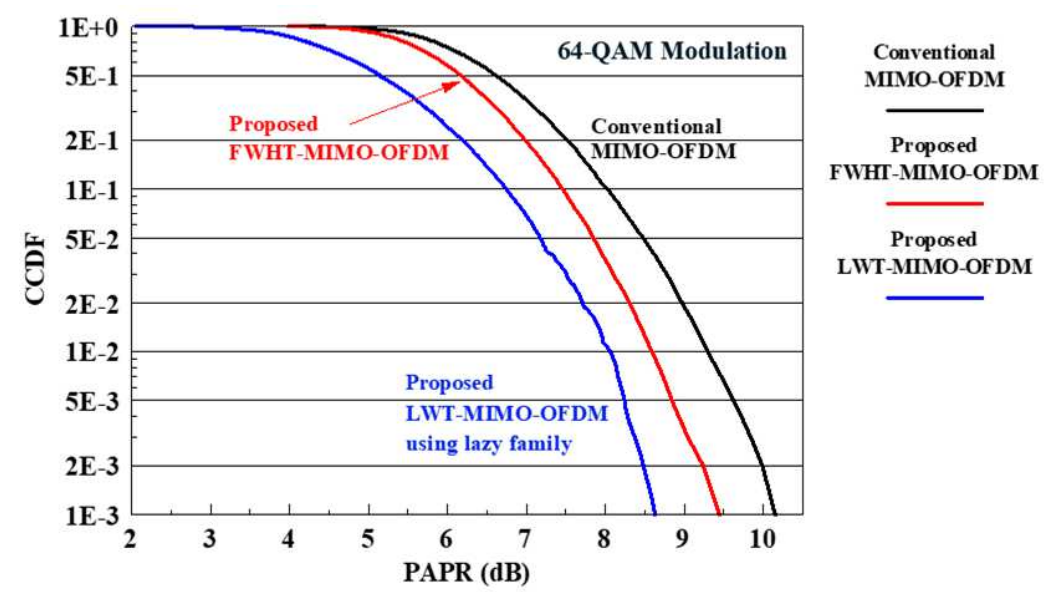

LWT-MIMO-OFDM

Fig. 10. Evaluation of PAPR accomplishments of classic MIMO-OFDM, suggested FWHT-MIMO-OFDM and recommended LWT-MIMO-OFDM systems for 64-QAM modulation.

Analyzing Figure 10, it is understood that similar outcomes and gains were acquired to the prior 16-QAM modulation.

\section{CONCLUSION}

In this article, it is proposed to combine LWT conversion and MIMO-OFDM system as a solution to PAPR problem, which is one of the biggest drawbacks of multi-input multi-output multi-carrier communication systems. In addition, the effects of FWHT conversion on the MIMO-OFDM system were investigated. While no contribution could be made to the BER performance with the proposed FWHT-MIMO-OFDM method, it was surprisingly observed that a PAPR gain of 1 dB was provided over the PAPR performance. However, the advantages of MIMO-OFDM systems such as high access quality, high data rates and spectral efficiency are further enhanced by the proposed LWT conversion. With the proposed LWT-MIMO-OFDM system, providing approximately $6 \mathrm{~dB}$ of SNR gain and approximately $2 \mathrm{~dB}$ of PAPR gain has revealed the importance of the study. Understanding that LWT conversion can be effective at these levels in MIMO-OFDM systems is the most important outcome of this article. From these inferences, it has been understood that LWT conversion is a very important method to be considered in $6 \mathrm{G}$ and beyond new generation communication technologies. 
Acknowledgements: This research was not funded.

Author's contributions: Author certifes that this material or similar material has not been and will not be submitted to or published in any other publication before. Furthermore, Author certifes that they have participated sufciently in the work to take public responsibility for the content, including participation in the concept, design, analysis, writing, or revision of the manuscript.

Availability of data and material: (data transparency) Based on the request.

Code availability: (software application or custom code) Not applicable.

Declarations Confict of interest: The author declares that they no confict of interest. The author of this research acknowledges that they are not involved in any financial interest.

\section{REFERENCES}

[1] R. Güngüneş, E. Tuna and M. Lüy, "Performance analysis of channel coding in multi input multi output orthogonal frequency division multiplexing", International Journal of Engineering Research and Development, vol. 10, no. 3, pp. 52-65, December 2018.

[2] A. Yavanoğlu and Ö. Ertuğ, "On the Capacity Analysis of Compact Space-Multimode Microstrip Antenna Arrays for IEEE802.11n MIMO-OFDM Systems using Sub-Optimal MIMO Detectors", IEEE 18th Signal Processing and Communications Application Conference (SIU 2010), pp.121-124, 2010.

[3] G.J. Foschini and M. Gans, "On limits of wireless communications in a fading environment when using multiple antennas", Wireless Personal Communications, vol. 6, pp. 311-355, March 1998.

[4] R. Prasad and R. V. Nee, OFDM for Wireless Multimedia Communications, Artech House Publishers, 2000.

[5] S. Çınar, Low-Complexıty Space-Tıme Block Coded OFDM Systems, Master Thesis, İstanbul Technical University, Graduate School of Natural and Applied Sciences, İstanbul, 2010.

[6] L. S. Gordon, R. B. John, W. M. Steve, L. Ye and A. I. Marry, "Broadband MIMO-OFDM Wireless Communications", Proceedings of the IEEE, vol. 92, No. 2, pp. 271-294, February 2004.

[7] K. Aksoy, High Performance Coding Techniques for MIMO Wireless Communıcation Systems Using OFDM Modulation, PhD. Thesis, İstanbul Technical University, Graduate School of Natural and Applied Sciences, İstanbul, 2008.

[8] A. Güner and A. Özen, "Lifting Wavelet Transform Based Multicarrier Modulation Scheme for Coherent Optical Communication Systems", Journal of Lightwave Technology, vol. 39, no. 13, pp. 4255-4261, July 2021.

[9] Y. İ. Tek, E. B. Tuna, A. Savaşcrhabeş and A. Özen, "A new PAPR and BER enhancement technique based on lifting wavelet transform and selected mapping method for the next generation waveforms", International Journal of Electronics and Communications, vol. 138, no. 8, pp. 1-14, published online 24 June 2021.

[10] M. K. Lakshmanan and H. Nikookar, "A review of wavelets for digital wireless communication", Wireless Personal Communications, vol. 37, no. 10, pp. 387-420, 2006.

[11] B. G. Negash and H. Nikookar, "Wavelet-based multicarrier transmission over multipath wireless channels", IEE Electronics Letters, vol. 36, no. 21, pp. $1787-1788,2000$.

[12] M. Jansen and P. Oonincx, Second GenerationWavelets and Applications, 2005.

[13] J. Maly and P. Rajmic, "Fast lifting wavelet transform and its implementation in java," in IFIP Int. Federation Inf. Process., Boston, MA, USA: Springer, pp. 488-496, 2007.

[14] M. Maraş, E. N. Ayvaz, M. Gömeç, A. Savaşcıhabeş and A. Özen, "A novel GFDM waveform design based on cascaded WHT-LWT transform for the beyond 5G wireless communications", Sensors, Vol. 21, No. 5, pp. 1-19, March 2021.

[15] C. Çiftlikli, M. Tören and A. Tuncay Özşahin "PAPR Reduction with Wavelet Transform and PTS Tecnique in MIMO-OFDM Systems", ELECO 2012 Elektrik - Elektronik ve Bilgisayar Mühendisliği Sempozyumu, pp. 350-354, 29 Kasım - 01 Aralık 2012, Bursa, Turkey.

[16] M. Tören, The Analysis of the Peak to Average Power Ratio (PAPR) Reduction in the Multiple-Input Multiple-Output Ortogonal Frequency Division Multiplexing (MIMO-OFDM) Systems, Master Thesis, Erciyes University, Graduate School of Natural and Applied Sciences, Kayseri, 2013.

[17] M. Tören and C. Çiftlikli, "Peak-to-Average-Power-Ratio (PAPR) reduction methods with wavelet transform in MIMO-OFDM", IETE Journal of Research, pp. 1-7, 2017.

[18] E. B. Tuna, Comparison of the Performances of MIMO-OFDM and Code Domain NOMA Systems, Bachelor Degree Dissertation, Nuh Naci Yazgan University, Kayseri, June 2020.

[19] N. Taşpınar and M. Yıldırım, "A Novel Parallel Artificial Bee Colony Algorithm and Its PAPR Reduction Performance Using SLM Scheme in OFDM and MIMO-OFDM Systems", IEEE Communications Letters, vol. 19, no. 10, pp. 1830-1833, October 2015. 